Purpose Three methods were considered for routine checking: UV spectrophotometry, flow injection analysis (FIA) and high performance liquid chromatography (HPLC).

Materials and Methods Three batches (3×200 capsules) were prepared with nystatin (INRESA) and mannitol (VWR). All other reagents were of analytical grade.

Preparation of stock solutions of nystatin and capsules content was in reagent-grade methanol for FIA and HPLC (nystatin $72 \mu \mathrm{g} / \mathrm{mL}$ ). For UV spectrophotometry, a subsequent dilution $(1 / 50$ $\mathrm{V} / \mathrm{V}$ ) with acetate ammonium buffer/methanol, 50:50 (V/V) was needed.

For FIA and HPLC, $10 \mu \mathrm{L}$ were injected. In all cases, absorbance was measured at $305 \mathrm{~nm}$.

UV spectrophotometry used a double beam spectrophotometer (UV $\mathrm{mc}^{2}$ - SAFAS)

FIA used an HPLC device (Ultimate 3000 - Dionex) in which the stationary phase was replaced by a capillary flow of water $(1.0 \mathrm{~mL} /$ $\left.\min ; 25^{\circ} \mathrm{C}\right)$.

HPLC equipment was an ELITE LaChrom (VWR/Hitachi). An end-capped $\mathrm{C} 18$ stationary phase was used $\left(30^{\circ} \mathrm{C}\right)$. The mobile phase was a mixture of $0.05 \mathrm{M}$ acetate ammonium buffer $\mathrm{pH}=6.0$ / reagent-grade methanol $(35 / 65 ; \mathrm{V} / \mathrm{V})$. Flow rate was $1.0 \mathrm{~mL} / \mathrm{min}$; run time was $25 \mathrm{~min}$.

Results For UV spectrophotometry and FIA, the development took into account the nystatin concentration to obtain absorbance levels suitable for the precision and the range of linearity. HPLC was developed as an isocratic stability indicating method.

The three methods were fully validated (ICH Q2R1). HPLC ruggedness was studied according the adjustments allowed by the $\mathrm{Ph}$. Eur. (2.2.46). Nystatin content (3 batches) assayed by each method complied with the acceptance limit: $90.0-110.0 \%$.

Conclusions For routine checking, UV spectrophotometry or FIA would be the methods of choice (rapid, easy to handle); the HPLC method could be used to perform stability studies.

No conflict of interest.

\section{TCH-007 DEVELOPMENT OF A BETAMETHASONE (DIPROPIONATE) TOPICAL EMULSION 0.1\% (W/W) (1MG/G) FOR CUTANEOUS T-CELL LYMPHOMA}

doi:10.1136/ejhpharm-2013-000276.198

P Trindade, ' $\mathrm{D}$ Mateus, ${ }^{1} \mathrm{H}$ Gonçalves, ${ }^{2} \mathrm{MF}$ Sachse, ${ }^{3 P} \mathrm{P}$ Machado, ${ }^{4} \mathrm{HM}$ Ribeiro, ${ }^{1} \mathrm{~A}$ Melo Gouveia. 'Instituto Português de Lisboa Francisco Gentil EPE, Pharmacy, Lisbon, Portugal; ' Instituto Português de Lisboa Francisco Gentil EPE, Dermatology, Lisbon, Portugal; ${ }^{3}$ Faculty of Pharmacy-University of Lisbon, Microbiology, Lisbon, Portugal; ${ }^{4}$ iMed.UL (Research Institute for Medicines and Pharmaceutical Sciences) FFUL, Nanomedicine and Drug Delivery Systems, Lisbon, Portugal

Background Cutaneous lymphomas are a heterogeneous group of lymphomas characterised by $\mathrm{T}$ and $\mathrm{B}$ clonal lymphoproliferative infiltrates that appear and remain confined to the skin without evidence of involvement of other organs/systems in the six months following diagnosis.

Some subtypes of cutaneous $\mathrm{T}$ epidermotropic lymphomas respond favourably to topical treatment with steroids. Betamethasone dipropionate is a synthetic high-potency glucocorticoid with anti-inflammatory and immunosuppressive action used as the main topical treatment in the early stages of LNH-T-Mycosis Fungoides, or as an adjuvant treatment in advanced stages of the disease.

In the Portuguese pharmaceutical market only a $0.05 \%(w / w)$ $(0.5 \mathrm{mg} / \mathrm{g}) \mathrm{cream}$ is available although for this therapeutic indication strengths in a range of $0.025 \%-0.1 \%(\mathrm{w} / \mathrm{w})$ are required. This was already an off-label clinical use and a higher concentration was required due to insufficient response to the concentration available. Purpose To prepare and characterise a topical 0.1\% (w/w) (1 mg/g) betamethasone (dipropionate) cream and evaluate the overall response in cutaneous T-cell lymphomas.
Materials and Methods Several batches of a compounded W/O emulsion containing betamethasone $0.1 \%(\mathrm{w} / \mathrm{w})(1 \mathrm{mg} / \mathrm{g})$ were prepared and analysed for macroscopic characteristics, $\mathrm{pH}$, rheological properties and microbiological quality (total germs, fungal, yeasts and E. coli)

Patients were evaluated monthly and the overall response was recorded (CR-cutaneous lesion totally disappeared; PR-partial remission - objective response $>50 \%<100 \%$; Stabilized disease if cutaneous lesions were similar; No response if cutaneous lesions worsened).

Results We obtained a white, homogeneous, opaque and odourless cream with a pseudoplastic behaviour. The $\mathrm{pH}$ of the formulations at $22 \pm 3^{\circ} \mathrm{C}$ was $5( \pm 0.5)$. Microbiological control for non-sterile products revealed no growth of micro-organisms.

By the end of the first month one patient (11.1\%) showed partial remission, the others $(88.9 \%)$ had their cutaneous disease lesions stabilised.

Conclusions The topical emulsion developed has $\mathrm{pH}$ values and rheological characteristics suitable for drug stability and topical skin application. Clinical data is still insufficient for any conclusions.

No conflict of interest.

\section{TCH-008 DEVELOPMENT OF A HYDROCHLOROTHIAZIDE $0.5 \mathrm{MG} / \mathrm{ML}$ ORAL SOLUTION FOR CHILDREN}

doi:10.1136/ejhpharm-2013-000276.199

YY Li. Royal Dutch Pharmacists Association, Scientific Institute of Dutch Pharmacists, Den Haag, The Netherlands

Background In The Netherlands there are no licenced medicines available with hydrochlorothiazide that are suitable for children. Lack of children's formulations in general may lead to a variety of mixtures of different quality and strength. This may cause medication errors, especially when children receive the same active substance in different formulations and strengths during their hospital stay and after discharge.

Purpose To develop a standardised hydrochlorothiazide liquid formulation with a shelf life supported by stability studies, in order to provide standardised and safe care for children.

Materials and Methods National standard procedures were applied to assess the therapeutic rationale and to design an oral solution for children. HPLC was used to develop a method of indicating stability in order to establish shelf life. A patient information leaflet was designed, also by following a standard procedure.

Nationwide, the quality of hydrochlorothiazide oral liquid preparations was determined pre- and post-introduction of the standardised formulation.

\section{Results}

- A therapeutic rationale was established for diuresis.

- A formulation for a robust hydrochlorothiazide $0.5 \mathrm{mg} / \mathrm{ml}$ oral solution was optimised for solubility, stability and taste.

- An HPLC method was developed to test stability.

- A shelf life of 6 months was established.

- Publication in the Dutch Formulary.

- A patient information leaflet was produced providing information on indication, use, precautions, interactions and storage.

- Hydrochlorothiazide formulation errors decreased nationwide from $35 \%$ to $13 \%$

Conclusions A robust and stable oral liquid formulation was developed containing hydrochlorothiazide $0.5 \mathrm{mg} / \mathrm{ml}$, which remains stable for 6 months. A patient information leaflet was made available. Standardization and publication in the Dutch Formulary has demonstrably improved the quality of hydrochlorothiazide oral liquid formulations nationwide.

No conflict of interest. 